\title{
Accurate Measurement of the Force Sensor for Intermediate and Proximal Phalanges of Index Finger
}

\author{
Nazrul H. Adnan \\ Advanced Intelligent \\ Computing and Sustainability \\ (AICoS) Research Group, \\ School Of Mechatronics \\ Engineering, Universiti \\ Malaysia Perlis (UNIMAP), \\ 02600 Arau, Malaysia
}

\author{
Khairunizam Wan \\ Advanced Intelligent \\ Computing and Sustainability \\ (AICoS) Research Group, \\ School Of Mechatronics \\ Engineering, Universiti \\ Malaysia Perlis (UNIMAP), \\ 02600 Arau, Malaysia
}

\author{
Shahriman AB \\ Advanced Intelligent \\ Computing and Sustainability \\ (AICoS) Research Group, \\ School Of Mechatronics \\ Engineering, Universiti \\ Malaysia Perlis (UNIMAP), \\ 02600 Arau, Malaysia
}

\begin{abstract}
In this research paper, the study for grip force on the maximum level of the various materials handling griper can be evaluate at an effective maximum isometric strength especially for intermediate and proximal phalanges of index finger. This analysis method using the piezoresistive force sensor, whereas the devices will be automatically increases the accuracy and repeatability of the force sensitivity. Force sensor is a component of flexible and easily applied to enable measurement of the non-intrusive value. The sensor can be attached to or placed on a variety of surface conditions. The physical structure of product is to be combined with plastic film or metal for increased stiffness or for added protection from abrasion. In order to determine forces acting upon an articular joint during fingers rehabilitation for maximum grip force on low cost DataGlove. The estimation show that all the action force is starting at their fingertips functioning as the total volume of gripper force, dimensions / orientation of the handle, and grip made. By measuring the gripper forces acting on the fingertips of several subjects, the different handle and level of gripper force are resulting from movement of fingers will be gathered and will be analyzed so that a realistic mathematical model structure could be produced.
\end{abstract}

\section{Keywords}

DataGlove; Fingers Force; Mathematical Model Structure; Piezoresistive Sensor; and Rehabilitation.

\section{INTRODUCTION}

The human fingers are one of the versatile and effective organs from the whole body and it's the primary tool by which humans manipulate object. For the example, the manipulation of grip force on the object is able to produce something to maintain and control such as the object handling. However Ayoub et al. and Drury [1,2] stated that when the diameter of the holder become larger the force will be decreasing, it is depending on the size of human / users hand. On the other hand, Johansson et al. [3] explained that when an object is compressed, it will result a force to be generated on the gripping finger. However, the grip of activities shall be accompanied by a force to prevent the compressed object from slipping. There are huge of research for analyze the finger force for example devices with the intelligent assistance [4], haptical interface devices [5,6], analysis of the human gripper force [7,8,9], instrumented intelligent gloves [10,11], footwear design [12], gesture recognition system [13], hemodynamic study [14], low cost DataGlove [15] and finally medical robotics. All of the researches are trying to seeking the best solutions for measuring the fingers force. Several methods can be done by using force sensor; according to $\mathrm{L}$. Paredes et al. [16] the best sensor devices for measuring the finger force should have the following list of the characteristics such as repeatability for measuring the high reading, a small physical size and light weight. Sensors are also required to increase the strength and reliability, a low drift rate, low cost, and may be a function at a high temperature and appropriate including the magnetic field [16].

The objective of this study is to finding the correlations relationship on the phalanges finger forces while performing at the most common activity and useful movements of the fingers grasping, in order to achieve an ideal force reduction of the complexity of the system. With the aim to obtain accurate results and affordable usage, GloveMAP is able to get the results and perform experiments on several tests perfectly. GloveMAP system is also able to record the movements as required.

In this study, several targets for accurate results have been targeted. One of the most is the minimum and maximum level of strength that can be done by several groups. For the examples: normal person (those who are able to use fingers and do grasp properly), the disabled person (people who are not able to use fingers and do grasp properly) and elderly person (people who have no ability as normal people to use fingers and do grasp properly). Lebossé et al. [18] stated in robotics or biomedical sector, the application of the force sensor or nonlinear sensors are able to be developed as the behavior modeling nonlinear low-cost force sensor.

For the mathematical model, the study will focus on the forces of the fingertips when the grasp activities are tested. At the same time the analysis using the probability will be considered. This research paper is structured as following section: Section 2 will be addresses as the literature reviews of the suitability researches, research approaches, research applications and research problems of identifying the grasping force. Section 3 will be describing some of the methodologies of the system. Section 4 will be describing the experimental design method including the experimental setup and result. Finally, Section 5 will present the conclusions and proposing some possible future work. 


\section{LITERATURE REVIEW}

The size and shape of the hand tool is one of the most important factors that affect usability. To ensure perfection in the interpretation of the diameter of the holder in determining the minimal grip force, time and tool holder will plays an important role. Each analysis conducted on the model will provide the estimations of the total force exerted on the fingertip grip force as a function of number, diameters of the holder, and the size of the user's/human hand $[1,2,20]$. Finger segments in the process of non-uniform distribution of grasping is proportional to the ability of finger showing a strong correlation with subjective assessment $[8,9,10,22]$. Human hand in the unparalleled ability to understand and manipulate objects, however, each researcher has tried to interpret but it still does not understand all the ramifications. Ayoub et al., Amis and Matei et al. [23,26,29] stated that the most valuable activities compare to the ancient (for example traditional robot hands) is about the grasping techniques, whereas it has many ways to interpret the movement of the finger grip including the ability to apply larger frictional forces.

Nowak et al. [30,31] said that the experiments of nine patients with the mixture between man and women according the different ages has been made using the a touch-digit grasping and the result shows that the moderate sensitivities between the patient can be made. Figure 1 shows the example of gripping techniques with the load lifting movement whereas the minimum force is required to preventing the slippery object.

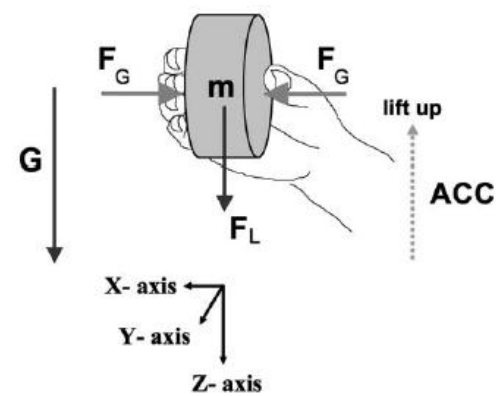

Fig 1. Example of how the cylindrical object with the hand / fingers configuration is used to do a grasping and lifting technique. The kinematic accelerations (ACC) will be functioning to the cylindrical object to measuring the finger forces (FG) and also the three others linear acceleration sensors. This activity is stated to measure in three dimensions axes (X, Y and $\mathrm{Z}$ ). The load force (FL) of the object's mass $(m=0.35 \mathrm{~kg})$ and the kinematic accelerations along the $\mathrm{Y}$ and $\mathrm{Z}$ axes including the gravity (G), $\left(\boldsymbol{F}_{L}=\mathbf{m} \times \sqrt{ }\left(\mathbf{G}+\boldsymbol{A C} \boldsymbol{C}_{Z}\right)^{2}+\sqrt{A C C^{2}}{ }_{Y}\right)[27]$

\section{METHODOLOGIES}

In translating the mathematical model for a fingers activity, it can be translated into a various models. System is unlimited to the dynamically systems only, but this system can be functioning into a statistical models, differential equations, or models of game theory. The definition of a mathematical model which is represented in many aspects, it is important by interpreting the existing system into a form which can be used [19]. There are six basic groups of variables including the variables of decision, input and state variables, exogenous and random variables, finally the output variables. In order to create a mathematical model equation by translate the gripping techniques; there are many factors that needed to be taken into account $[19,20]$. For an example the dimensional of the homogeneity requirement and information of quantitative, both of the following factors are closely related to influencing parameter for mathematical model factors. Jouni et al. [20] stated that the grip force of a typical finger $f^{(i)}-\left({ }^{\prime} i\right.$ ' $=$ little ring, middle or index fingers). On the other hand Langhaar [21] stated that the single finger grip force $f=\sum f^{(i)}$, hand $l$ and handle diameter $D$ can be used in determine the normal problems based on the dimensional homogeneity with a valid equations of the physics. It gives that the distribution of forces must be in the form of Eq. 1, in which $\alpha^{i}$,s are to be found experimentally.

$$
\frac{f^{i}}{f}=\alpha^{i}\left(\frac{D}{l}\right),
$$

\subsection{Total Grip Force}

In order to clarify the sensor characteristics, the sum of the energy can be obtaining by summing up all the 16 finger sensors that are attached at the individual hands, $F_{i j}$ is the force segment ( $\mathrm{i}=1$; index; 2 , middle; 3 , ring; 4 , little; and $\mathrm{j}=$ 1; distal; 2, middle; 3, proximal; 4, metacarpal) [22].

$$
F_{\text {total }}=\sum_{j=1}^{4} \sum_{i=1}^{4}\left[F_{i j}\right]
$$

To clarify the mathematical formula for the method used in this study are as follows by summing the 2 individual sensor of index and middle fingers of 5 respondents :-

$$
F_{\text {total }}=\sum_{j=1}^{4} \sum_{i=1}^{2}\left[F_{i j}\right]
$$

\subsection{Finger Forces Distribution}

Jouni et al. [20] stated that the force distributions on the gripping activities cannot be depending only on the gripping force $f$, if the selected independent variables are influencing the formation, the affecting quantity of maximal grip force $\bar{f}$ are the maximal grip force over the possible diameters of the handle (maximum of the maxima) $\bar{f}_{\text {opt }}$. The corresponding diameter $D_{o p t}$, and the maximal muscle force for very large diameter $\mathrm{D} \rightarrow \infty$ denoted by $\bar{f}_{\text {lim }}$ and argument based on dimensional homogeneity in which $\beta$ is a function to be found by experimentally approach [20].

$$
\frac{f}{f_{o p t}} \leq \frac{\bar{f}}{f_{o p t}}=\beta\left(\frac{\bar{f}_{\text {lim }}}{\bar{f}_{\text {opt }}}, \frac{D}{D_{\text {opt }}}\right) \approx \varepsilon \frac{\varepsilon \delta+2(1-\delta)}{\varepsilon^{2}+1-\delta},
$$

The rational approximation of $\beta$ on the right hand side of Eq 4 defined by $\delta=\bar{f}_{\text {lim }} / \bar{f}_{\text {opt }}$ and $\varepsilon=D / D_{\text {opt }}=(D / l) /\left(D_{\text {opt }}\right.$ (l) $=k / k_{\text {opt }}$, the maximal force is zero at $k=0$ and the maximum of the maxima is attained at $k_{\text {opt }}$. For the maximal force takes the limit value $\bar{f}_{\text {lim }}$ when $k \rightarrow \infty 1$ (corresponds to the pressing of a plane with the fingertips). For the other fingers, it's separately with obvious modifications i.e $l \rightarrow l^{i}$, etc. By the mathematical definitions, the parameter physical meanings of $\delta$ and $\varepsilon$ are the set of the ratio of maximal muscle force [20]. Ayoub et al. [1] also stated that that maximal fingertip force can be used to calculate the amount of distal phalanx forces and the distribution of powers as a function of diameter at maximum energy level [23]. 

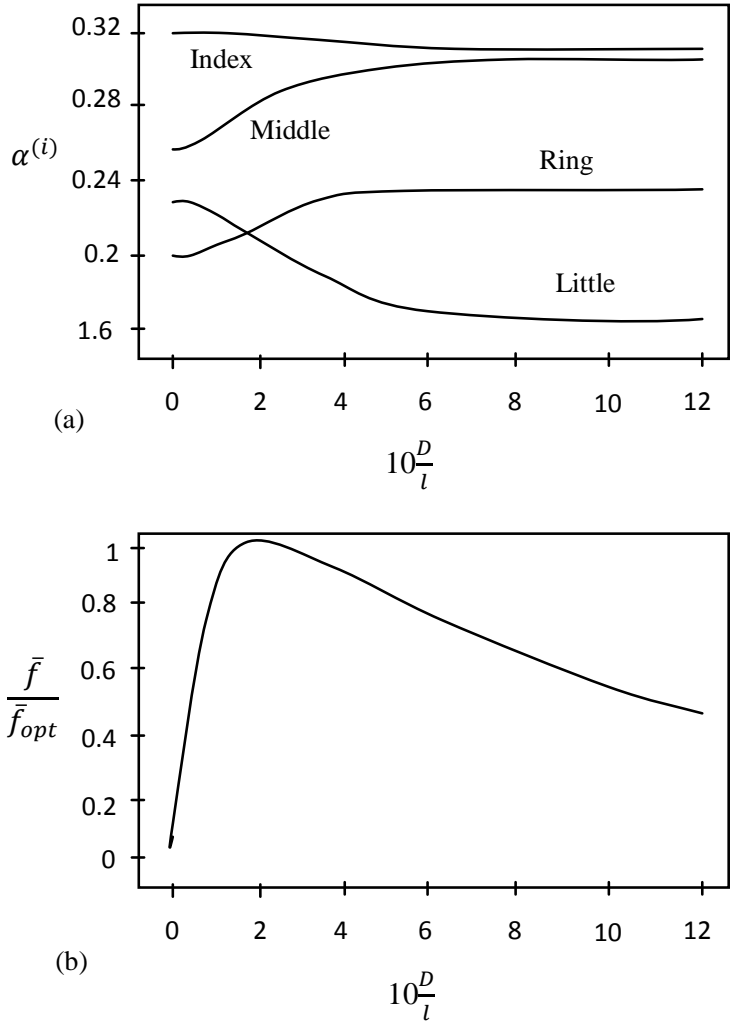

Fig 2: Distribution of distal phalanges in the gripping object as a function of the cylinder diameter [20].

Figure 2 show the handle diameter $D$, middle finger length $l$ and $\bar{f}_{\text {opt }}$ the maximal grip force and reconstructed using Eq. 4 with the measuring values of the index and middle distal phalange, respectively [20].

\subsection{The Distribution Model of Forces}

The basic corresponding parameters for non-linear leastsquares fitting can be obtained by Eq. 5 . Therefore, the model that describing the distribution of forces is given by:-

$$
\begin{gathered}
\frac{f}{f_{o p t}} \leq \frac{\bar{f}}{\bar{f}_{o p t}}=\varepsilon \frac{\varepsilon \delta+2(1-\delta)}{\varepsilon^{2}+1-\delta} \\
\frac{f^{(i)}}{f}=\alpha^{i}
\end{gathered}
$$

\section{EXPERIMENTAL DESIGN METHOD}

Various processes can be done to translate each experiment or test which was carried out. To transform the results into a realistic, the whole process will go through the experiment which is called as a design of experiments, or experimental design, (DoE). DoE can be related to the uniqueness of any information involving the analysis of probability exercises however, in controlled experiments DoE are often used to solving some of the difficulties in the analytical design.

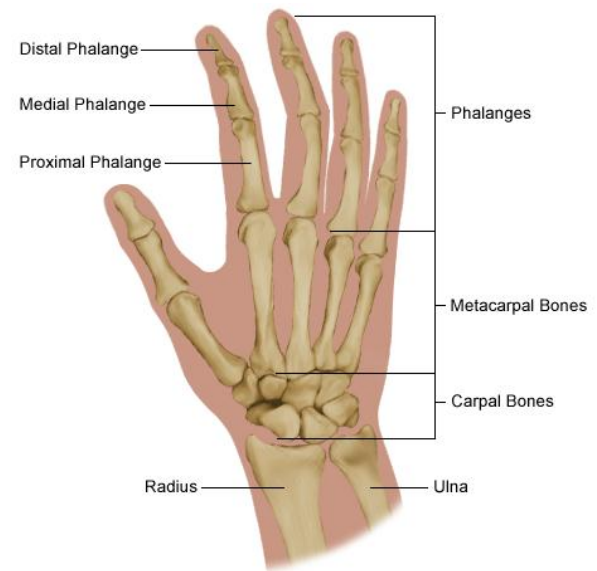

Fig 3: Anatomy of the hand [32]

$$
\begin{array}{r}
\sum_{i=1}^{a} \sum_{j=1}^{b} \sum_{k=1}^{c}\left(Y_{i j k}-\bar{Y} \ldots\right)^{2}=r . b \cdot \sum_{i=1}^{a}\left(\bar{Y}_{i . .}=-\bar{Y} \ldots\right)^{2}+r \cdot a \cdot \sum_{j=1}^{3}\left(\bar{Y}_{j}-\bar{Y} \ldots\right)^{2} \\
+r \times \sum_{i=1}^{a} \sum_{i=1}^{b}\left(\bar{Y}_{i j}-\bar{Y}_{i} . .-\bar{Y}_{j}+\bar{Y}\right)^{2}+\sum_{i=1}^{a} \sum_{i=1}^{b} \sum_{k=1}^{r}\left(Y_{i j k}-\bar{Y}_{i j}\right)^{2}
\end{array}
$$

Analysis of variance (ANOVA) is a sum set of the statistical models collection, in which functioning to observed the variance in certain variables and the statistical formula are divided into several components that be able to attributable in the different sources of variation. In a simplest form, ANOVA also able to provides a significant test whereas several groups are equal. For this reason, ANOVAs statistical analyses are much useful in comparing more than two, three, or more means [24].

Table 1. ANOVA List of Table [24]

\begin{tabular}{ccccc}
\hline Source & $\begin{array}{c}\text { Degrees of } \\
\text { Freedom }\end{array}$ & $\mathrm{SS}$ & $\mathrm{MS}$ & $\mathrm{F}$ \\
\hline A & $\mathrm{a}-1$ & $S S_{A}$ & $M S_{A}$ & $M S_{A} / M S_{\text {within }}$ \\
B & $\mathrm{b}-1$ & $S S_{B}$ & $M S_{B}$ & $M S_{B} / M S_{\text {within }}$ \\
A X B & $(\mathrm{a}-1)(\mathrm{b}-1)$ & $S S_{A X B}$ & $M S_{A X B}$ & $M S_{A X B} / M S_{\text {withi }}$ \\
Within & $\mathrm{ab}(\mathrm{r}-1)$ & $S S_{\text {within }}$ & $M S_{\text {within }}$ & \\
\hline Total & $\mathrm{abr}-1$ & $S S_{\text {total }}$ & & \\
\hline
\end{tabular}

\subsection{Experimental Setup}

Flexible sensors or flex sensor is a resistor that is actually made up of small spots of carbon. Flexible sensor applications will make the system changes the resistance when the physical of the flex sensor is bent. It is known as the ideal input device for controlling the mechanism due to easy to position when attached to the user fingers [25].

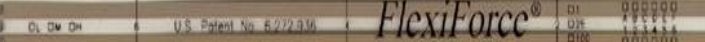

Fig 4: Flexiforce Pressure Sensors [25]

\subsubsection{Processing of the Force Data Collection}

Before the process of measuring the finger force, the human fingers fall into several stages, it is known as the middle, index, ring and little finger. On each finger there are 3 parts or segments in which it is known as the distal phalanges, 
intermediate phalanges, and proximal phalanges as shown in Fig 5, these segments will be assessed for each grip force. Figure 6 show the example of heptic glove constructs and built by L. Paredes et al. [16]. Location of this sensor is placed under the finger where it is intended to ensure that the reaction force will be more easily interpreted through several stages that have been clarified. By using this force sensor, it can translate the individual ability to do the grip of an object.

Figure 6 shows that the suitable location that a FlexiForce sensor is being installed on the palm side of the GloveMAP, in this fabrication the Integrated Microprocessor Unit (IMU) / Arduino Processor Unit (APU) and the Finger Flexion Sensors (FFS) / Flexible Bend Sensor are ideally to be installed on the dorsal-side of the glove [17].

\subsection{Experimental Result}

The resulting data were obtained from five test sessions. Each of tests comprises by two flexible force components that were located at index finger and middle finger. All tests conducted on five respondents will be average and evaluated against the statistical model (ANOVA). Two factors of ANOVA value was used in the analysis which is factor 1 stated as a length of respondent finger and factor 2 is handle diameter. The process results will be further analysis and justification research of GloveMAP, low cost measurement glove.

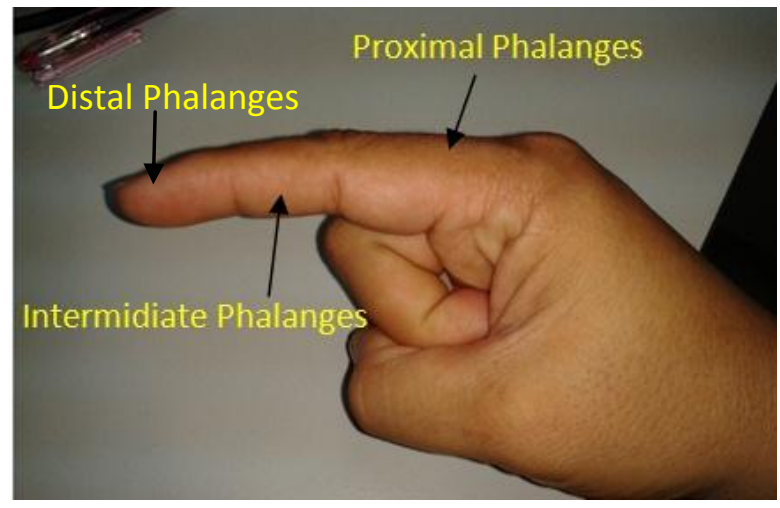

Fig 5: Mapping joint of human fingers

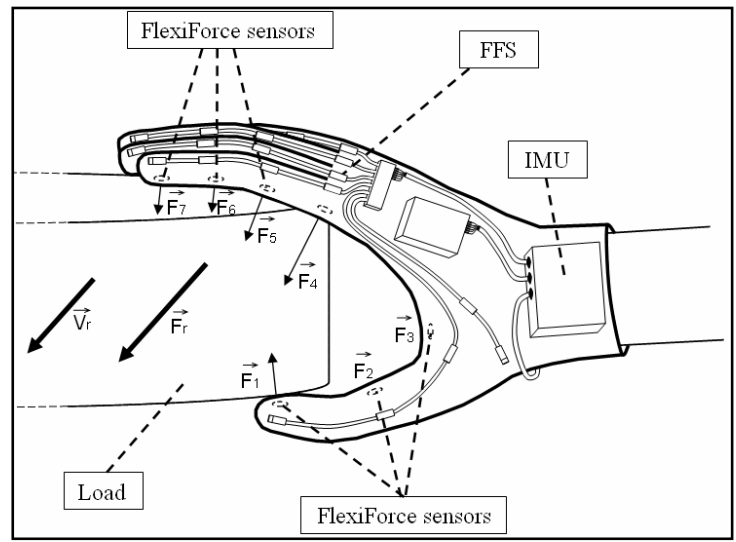

Fig 6: Sketch of the haptic glove under development [16].

The test respondent's finger force corresponding to subject were calculated and placed into the table of the analysis of variance (ANOVA) (Table 2). These data were used to verify the force measurement value (Response 1 and Response 2) which is related with the corresponding of the finger length and the diameter of the material.

\subsubsection{Individual Phalange Force}

The ANOVA summary for selected factorial model Table 3 and Table 4 shows that finger length and handle diameter of GloveMAP from five tested respondents are significantly at $\mathrm{P}$ $\geq 0.05$ levels of significance for Response 1 . The "Lack of Fit F-value" of 16.13 are implies at the Lack of Fit is slightly significant. There is only a $1.06 \%$ chance that a "Lack of Fit F-value" could occur due to noise. Meanwhile for Response 2 as shown in Table 4, the statistically data are not significant at the $\mathrm{P}<0.05$ level of significance.

\subsection{Maximal Fingers Forces and Handle Diameter}

Amis [26] stated that the study on effect of maximal gripping based on the handle diameter and fingertips forces has been studied. According to these data Fig 7 the maximal force value for both force sensors are increasing to the peak value of the graph. On the other hand, the force will be decreased when there is no force on the fingers and the value reach to the minimum value of force. The maximal force must be taken a maximal value of the griper value with some value of the handle diameter.

Table 2. The list of ANOVA result that relatively to the finger forces factors and some responses when the force level is taken into the respondent fingers.

\begin{tabular}{|r|r|r|r|r|r|r||}
\hline Std & Run & Block & $\begin{array}{c}\text { Factor 1 } \\
\text { A:Finger Length } \\
\text { MM }\end{array}$ & $\begin{array}{c}\text { Factor 2 } \\
\text { B:Handle Diameter } \\
\text { MMM }\end{array}$ & $\begin{array}{c}\text { Response 1 } \\
\text { Force 1 } \\
\text { Newtons (N) }\end{array}$ & $\begin{array}{c}\text { Response 2 } \\
\text { Force 2 } \\
\text { Newtons (N) }\end{array}$ \\
\hline 10 & 1 & Block 1 & 84.00 & 40.00 & 5.5 & 5.36 \\
\hline 3 & 2 & Block 1 & 65.00 & 40.00 & 5.47 & 5.36 \\
\hline 4 & 3 & Block 1 & 65.00 & 40.00 & 5.52 & 5.35 \\
\hline 18 & 4 & Block 1 & 84.00 & 60.00 & 5.72 & 5.38 \\
\hline 11 & 5 & Block 1 & 65.00 & 60.00 & 5.92 & 5.36 \\
\hline 16 & 6 & Block1 & 84.00 & 60.00 & 6.04 & 5.32 \\
\hline 17 & 7 & Block1 & 84.00 & 60.00 & 6.07 & 5.35 \\
\hline 15 & 8 & Block 1 & 65.00 & 60.00 & 5.91 & 5.36 \\
\hline 1 & 9 & Block 1 & 65.00 & 40.00 & 5.6 & 5.36 \\
\hline 14 & 10 & Block 1 & 65.00 & 60.00 & 5.51 & 5.35 \\
\hline 20 & 11 & Block 1 & 84.00 & 60.00 & 5.5 & 5.34 \\
\hline 9 & 12 & Block 1 & 84.00 & 40.00 & 5.51 & 5.75 \\
\hline 12 & 13 & Block 1 & 65.00 & 60.00 & 5.51 & 5.92 \\
\hline 2 & 14 & Block 1 & 65.00 & 40.00 & 5.52 & 6.17 \\
\hline 13 & 15 & Block 1 & 65.00 & 60.00 & 5.51 & 5.8 \\
\hline 7 & 16 & Block1 & 84.00 & 40.00 & 5.52 & 5.78 \\
\hline 8 & 17 & Block1 & 84.00 & 40.00 & 5.52 & 5.73 \\
\hline 6 & 18 & Block 1 & 84.00 & 40.00 & 5.53 & 5.92 \\
\hline 5 & 19 & Block 1 & 65.00 & 40.00 & 5.48 & 5.72 \\
\hline 19 & 20 & Block 1 & 84.00 & 60.00 & 5.53 & 5.33 \\
\hline & & & & & & \\
\hline
\end{tabular}

Table 3. ANOVA summary table for Response 1

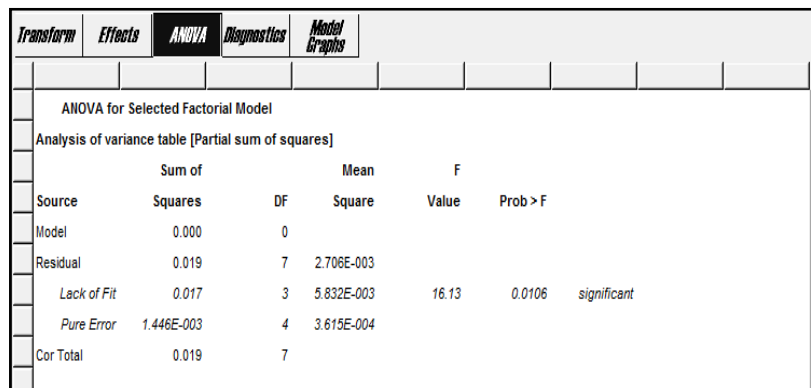


Table 4. ANOVA summary table for Response 2

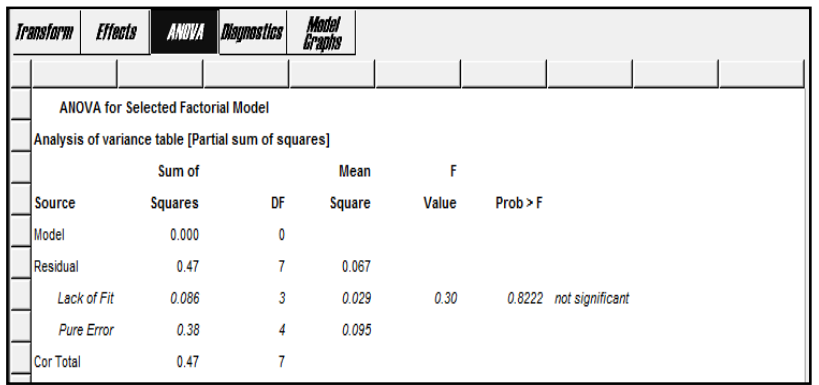

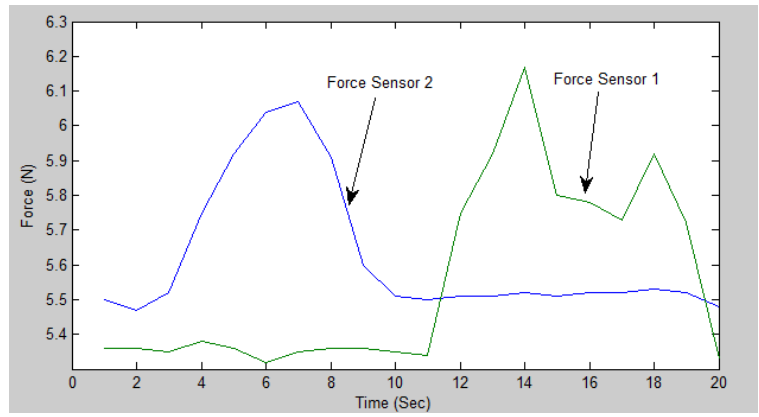

Fig 7: Maximal bending force for sensor 1 and sensor 2

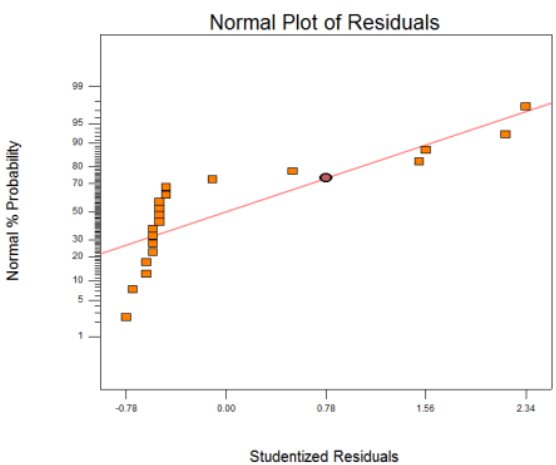

(a)

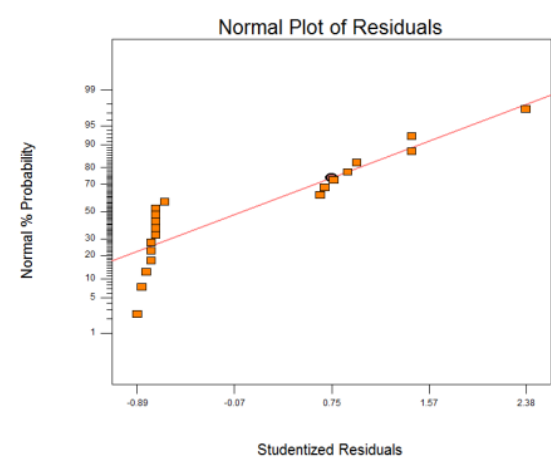

(b)

Fig 8: The relative normal plot of residuals (a) Force Sensor 1 (b) Force Sensor 2 when the grasping of the cylindrical material prediction by the ANOVA

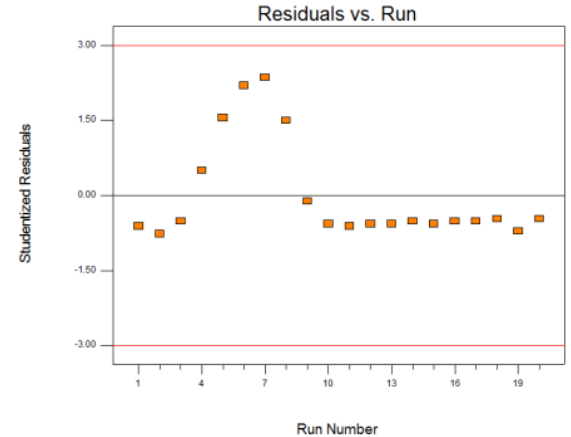

(a)

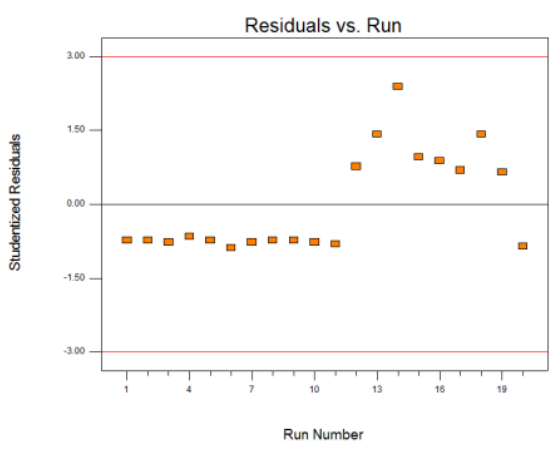

(b)

Fig 9: The distributions of the residuals vs. run (a) Force Sensor 1 (b) Force Sensor 2 during the grip of the cylindrical material /object

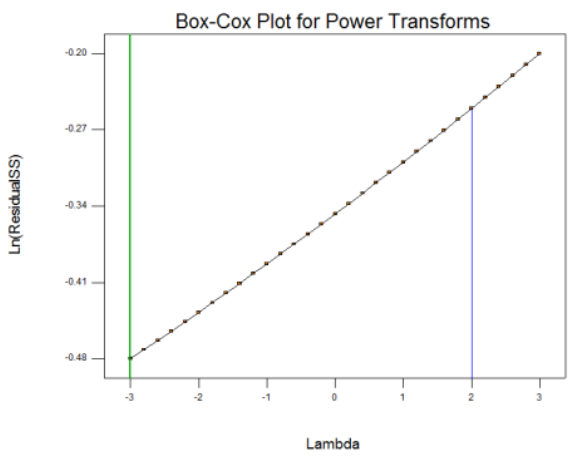

(a)

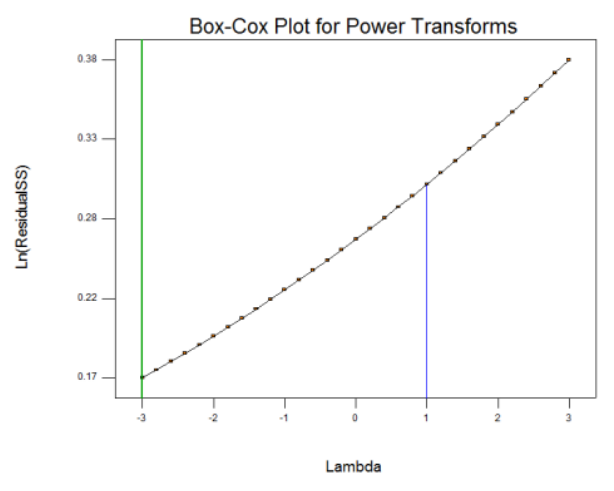

(b)

Fig 10: The Box-Cox Plot for power transforms (a) Force Sensor 1 (b) Force Sensor 2 during the grip of the cylindrical material / object 
Table 5. Recommended handle diameters for maximizing subjective comfort [22]

\begin{tabular}{|c|c|c|c|c|}
\hline \multirow[t]{2}{*}{ Hand size } & \multicolumn{2}{|l|}{ Female } & \multicolumn{2}{|l|}{ Male } \\
\hline & Hand Length (mm) & Handle Diameter (mm) & Hand Length (mm) & Handle Diameter (mm) \\
\hline Small & $160.0-169.8$ & 31.5-33.4 & 175.0-186.0 & 34.4-36.6 \\
\hline Medium & 169.8-180.3 & 33.4-35.5 & 186.0-196.3 & 36.6-38.6 \\
\hline Large & 180.3-190.0 & 35.5-37.4 & 196.3-205.0 & $38.6-40.3$ \\
\hline
\end{tabular}

To clarify the use of force in respect of the ANOVA analysis, the first force sensor is significant compared with the second force sensor. This is because the pressure or force exerted on the index finger is more pressure / force against the force sensor located on the middle finger. This occurs when there is an activity of grasping on the material, so that the pressure will be more focused on targeted finger.

Figure 8, Figure 9 and Figure 10 shows that the individual force ware defined as the total sum of the fingers movement of the four phalange segments. The fingers and phalanges forces are the main parts in controlling the handle. Yong-Ku Kong et al. [22] stated the contribution average the total grip finger force was the highest (34.8\%), followed by the ring total gripping (26.5\%), index and little fingers (24.9\%) and $(13.8 \%)$. Table 5 stated that an average of $41.6 \%$ of total grip finger force was exerted by the distal phalanges, $23.7 \%$ by the middle phalanges, $19.0 \%$ and $15.7 \%$ by the proximal and metacarpal phalanges, respectively [22].

\section{CONCLUSION}

An analytical mathematical model and analysis of variance (ANOVA) has been established to predict the force induced at the flexible force sensor and the human finger of low cost DataGlove. In this paper, the proposed wearable DataGlove rehabilitation device equipped with two force sensors was developed. The main objective of this study is to shows that the fingers power grip will exert more during the grasping task. The resultant of the experiment showed a good agreement of theoretical modeling results plus the experimental results for two flexible force sensor samples and a human finger stimulated with a variable range of input voltages. Five testers or candidates have been used to find the best force in reviewing the appropriateness analysis. In other hand, the analysis also focusing on the suitability force sensor to test the ability of DataGlove to be translated in the application that will be planned in future projects. For future work, the project involves on further enhancing the capability of sensory unit by designing and analyzing the low cost DataGlove especially for the Rehabilitation and Therapeutic Health System.

\section{ACKNOWLEDGMENTS}

Special thanks to all members of UNIMAP Advanced Intelligent Computing and Sustainability (AICoS) Research Group and School Of Mechatronics Engineering, Universiti Malaysia Perlis (UNIMAP), 02600 Arau, Malaysia for providing the research equipment and internal foundation. This work is supported by the Portable Research Grant Scheme by the Ministry of Higher Education Malaysia to Universiti Malaysia Perlis (PRGS 9013-00004).

\section{REFERENCES}

[1] Ayoub MM, Presti PL 1971. The determination of an optimum size cylindrical handle by use of electromyography. Ergonomics; 4:503-18.
[2] Drury CG 1980. Handles for manual materials handling. Appl Ergon; 1:35-42.

[3] Johansson, R.S., Ha“ger, C., Ba“ckstro"m, L. Somatosensory 1992. Control of precision grip during unpredictable pulling loads III. Impairments during digital anaesthesia. Exp. Brain Res. 89, 204-213.

[4] D. McGee, P. Swanson March 2001. Method of Controlling an Intelligent Assist Device, U.S. Patent No. $6,204,620$.

[5] M. Monroy, M. Ferre, J. Barrio, V. Eslava, I. Galiana, April 2009. Sensorized Thimble for Haptics Applications, in IEEE International Conference on Mechatronics. (Málaga, Spain), pp.1-6.

[6] Z. Ye, G. Auner, 2003. Haptic Interface Prototype for Feedback Control on Robotic Integration of Smart Sensors, in IEEE International Conference on Control Application, pp. 995-1000.

[7] K. N. Tarchanidis, J.N. Lyngouras, June 2003. DataGlove With a Force Sensor, in IEEE Transactions on Instrumentation and Measurement, vol. 52, pp. 984-989.

[8] M.C.F. Castro, A. Cliquet Jr, June 1997. A Low-Cost Instrumented Glove for Monitoring Forces During Object Manipulation, in IEEE Transactions on Rehabilitation Engineering, vol. 5, pp. 140-147.

[9] J-H. Lee, Y-S Lee, S-H Park, M-C Park, B-K Yoo, S-M In, October 2008. A Study on the Human Grip Force Distribution on the Cylindrical Handle by Intelligent Force Glove (I-Force Glove), in International Conference on Control, Automation and Systems.(Seoul, Korea), pp. 966-969.

[10] H. Kazerooni, D. Fairbanks, A. Chen, G. Shin, April 2004. The Magic Glove, in IEEE International Conference on Robotics and Automation, (New Orleans, LA), pp. 757-763.

[11] L. Dipietro, A. M. Sabatini, P. Dario, July 2008. A Survey of Glove-Based Systems and their Applications, in IEEE Transactions on Systems, Man, and Cybernetics, vol. 38 , pp. 461-482.

[12] J. H. Ahroni, E. J. Boyko, R. Forsberg, October 1998 Reliability of F-Scan In-Shoe Measurements of Plantar. Pressure in Foot and Ankle International, 9, 10 pp. 668673.

[13] Mohd Azri ABD AZIZ, Khairunizam WAN, Shahriman A.B, Siti Khadijah ZA'ABA and Shamsul Azri MOHD NOR 2011. An Adaptive Gesture Recognition System: Body Structure Cue; International Postgraduate Conference (IPCE).

[14] Shahriman A.B., M.N. Rahman Y., Siti Khadijah ZA'BA, Khairunizam WAN, Abdul Halim ISMAIL, Khairul A.H., and SA Roohi 2012. Hemodynamic Study On Upper Extremity: Simulation On Straight Reverse Saphenous 
Vein Graft; International Conference of Biomedical Engineering (ICoBE).

[15] Nazrul Hamizi Adnan, Khairunizam WAN, Shahriman A.B., Siti Khadijah ZA'BA, Hazry Desa, and Mohd Azri ABD AZIZ 2012. The Development of a Low Cost DataGlove by Using Flexible Bend Sensor for Resistive Interfaces; 2nd International Malaysia-Ireland Joint Symposium on Engineering, Science and Business (IMiEJS)

[16] L. Paredes-Madrid, P. Torruella, P. Solaeche, I. Galiana and P. Gonzalez de Santos, 2010. Accurate modeling of low-cost piezoresistive force sensors for haptic interfaces, in IEEE International Conference on Robotics and Automation, May 3-8, pp 1828-1833.

[17] Information on http://www.cytron.com.my /datasheet/sensor /FLXFC.pdf

[18] C. Lebossé, B. Bayle, M. de Mathelin, P. Renaud, May 2008. Nonlinear modeling of low cost force sensors, in IEEE International Conference on Robotics and Automation, (Pasadena, CA, USA), pp. 3437-3442.

[19] Information on http://www.sciencedaily.com /articles/m /mathematical_model.htm

[20] Jouni Freund, Risto Toivonen and Esa-Pekka Takala 2002. Grip forces of the fingertips, in Clinical Biomechanics 17 pp. 515-520.

[21] Langhaar HL, 1980. Dimensional Analysis and Theory of Models.Huntington, NY: Krieger.

[22] Yong-Ku Kong and Brian D. Lowe, 2005. "Optimal cylindrical handle diameter for grip force tasks", in International Journal of Industrial Ergonomics 35 495507.
[23] Ayoub MM, Presti PL, 1971. The determination of an optimum size cylindrical handle by use of electromyography. Ergonomics;4:503-18.

[24] Information on http://en.wikipedia.org/wiki /Analysis_of_variance

[25] Information on http://www.tekscan.com/flexible-forcesensors

[26] Amis AA, 1987. Variation of finger forces in maximal isometric grasp tests on a range of cylinder diameters. J Biomed Eng; 9:313-20.

[27] Angela Didomenico and Maury A. Nussbaum, 2003 Measurement and prediction of single and multi-digit finger strength; Ergonomics, vol. 46, no. 15, 1531 - 1548

[28] Angela DiDomenico Astin 1999. Finger force capability: measurement and prediction using anthropometric and myoelectric measures. Master of Science Thesis.

[29] Matei Ciocarlie, Andrew Miller and Peter Allen. Grasp Analysis Using Deformable Fingers. National Science Foundation under Grant No. IIS-03-12693

[30] Dennis A. Nowak and Joachim Hermsdörfer, 2003 Selective deficits of grip force control during object manipulation in patients with reduced sensibility of the grasping digits; Neuroscience Research 47 (65-72)

[31] Nowak, D.A., Hermsdörfer, J., Marquardt, C., Topka, H., 2002. Moving objects with clumsy fingers: how predictive is grip force control in patients with impaired manual sensibility. Clin. Neurophysiol. 114, 472_ 487.

[32] Information on http://www.yalemedicalgroup.org/stw/ Page.asp?PageID=STW023547 